Journal of Advanced Research in Fluid Mechanics and Thermal Sciences

Journal homepage: www.akademiabaru.com/arfmts.html ISSN: 2289-7879

\title{
Experimental Investigation of the Effect of a Downstream Square Plate on Vortex-induced Vibration and Galloping of a Square Cylinder
}

\author{
Azhar Mohamed ${ }^{1}$, Sheikh Ahmad Zaki ${ }^{1,}{ }^{*}$, Masataka Shirakashi ${ }^{2}$, Mohamed Sukri Mat Ali ${ }^{1}$, \\ Muhammad Zulfahmi Samsudin ${ }^{3}$, Ahmad Faiz Mohammad ${ }^{1}$ \\ 1 Malaysia-Japan International Institute of Technology, Universiti Teknologi Malaysia, Jalan Sultan Yahya Petra, 54100 Kuala Lumpur, Malaysia \\ Nagaoka University of Technology, Kamitomioka 1603-1, Nagaoka, Niigata, Japan \\ 3 Mechanical Engineering Section, Science and Engineering Department, Malaysia Japan Higher Education Program (MJHEP), Lot 2333 Jalan Kajang \\ Seremban, 43700 Beranang, Selangor, Malaysia
}

\section{ARTICLE INFO}

\section{Article history:}

Received 26 December 2019

Received in revised form 27 January 2020

Accepted 28 January 2020

Available online 25 March 2020

\section{Keywords:}

Square cylinder; square plate; vortexinduced vibration; galloping; vibration control
ABSTRACT

\begin{abstract}
A square cylinder exposed to a fluid flow is regarded as the simplest model of the structures in sea water or air flow, and known to show the Karman vortex induced vibration (KVIV) and the galloping in separate or overlapping ranges of flow velocity. In this study, wind tunnel experiments were performed on the cross-flow vibration of a square cylinder, and effects of a downstream square plate on the vibration were investigated to develop a convenient passive technique for vibration control. The square plate has a side length $w$ equal to the side length of the square cylinder $D(=26$ $\mathrm{mm}$ ) and set downstream with the gap ratio $S / D$ in the range of 0.12 to 2.90 where $S$ is the separation gap. The results show that the square plate has only little influence on KVIV and enhances the galloping, resulting in the lower critical reduced velocity of galloping $U_{g}$ i.e. the onset velocity of galloping reduced to about half of the isolated counterpart at $S / D=0.98$. Most remarkable effect of the downstream square plate is the enhancement of the vibration near the peak velocity of KVIV at $S / D=1.17$, which is around five times larger as compared with the isolated counterpart. This may

indicate occurrence of the wake-body-interference fluid elastic vibration.
\end{abstract}

Copyright $@ 2020$ PENERBIT AKADEMIA BARU - All rights reserved

\section{Introduction}

The study of flow induced vibrations (FIVs) of a cylindrical body exposed to flow is important in many engineering fields including aeronautical, offshore, civil and mechanical. FIV has caused tremendous structural damage such as the Tacoma Narrows bridge collapse in 1940, sodium leak and fire of the Monju fast breeder reactor in December 1996 and the oscillation observed for the

\footnotetext{
* Corresponding author.

E-mail address: sheikh.kl@utm.my (Sheikh Ahmad Zaki)
}

https://doi.org/10.37934/arfmts.68.1.98113 
Storebaelt East Bridge in Denmark, May 1998 (Terres-Nicoli and Kopp [1]). Hence, numerous studies have been devoted to clarify the FIV mechanisms and to predict and control the FIVs of cylindrical bodies, mainly the circular cylinder as it is most significant from the view point of fluid mechanics and it is commonly used in engineering applications.

One of the passive techniques proposed so far to suppress the vibration is the near-wake-body method which is advantageous since neither additional body touching the vibrating structure nor change of the supporting structure is required. In this method, other bodies are positioned downstream of the oscillating cylinder, e.g. splitter plates (Assi et al., [2]; Ali et al., [3]; Maruai et al., [4]; Dash et al., [5]), a circular cylinder (Shirakashi et al., [6]; Bae et al., [7]; Ngyuen et al., [8]; Ma et al., [9]; Chauhan et al., [10]) or a strip plate in cruciform arrangement (Koide et al., [11]; Kato et al., [12]; Kawabata et al., [13]) and fairings (Assi et al., [14]; Yu et al., [15]; Wang et al., [16]; Lou et al., [17]; Zheng and Wang [18]).

Two different cross-flow vibrations, the Karman Vortex Induced Vibration (KVIV) and the galloping, are well known for cylindrical bodies with the non-circular cross section. KVIV is a resonance-like vibration excited by the alternating lift force due to the periodic shedding of Karman vortex (KV) which exists if the cylinder is at rest. Synchronization, often called "lock-in", of the vortex shedding with the oscillation gives a non-linear character to KVIV, making its prediction extremely difficult. The galloping is caused for cylinders with the non-circular cross section due to the instability of a fluid- structure system. The vibration is driven by the time-averaged fluid force which develops in a quasi-steady flow relative to the oscillating body.

A square cylinder is regarded as the simplest model of the non-circular cross section structures, and known to show KVIV and galloping in separate or overlapping ranges of flow velocity, depending on the condition of the system. Koide et al., [11] studied the influence of a long strip plate with a width $w$ on the vibration of a square cylinder with a side length $D$, where the strip plate is set downstream in cruciform arrangement with a gap $S$. They reported that a strip plate with a width ratio $W / D=1$ effectively suppresses $K V I V$ at $S / D<2$ and galloping at $S / D<3.8$. However, a new vibration induced by periodic shedding of longitudinal vortices near the cross, known as the longitudinal vortex induced vibration (LVIV), was found to occur over a certain velocity range when $S / D$ is around 1.4 .

The investigation on the wake body as a passive control is still limited, especially on a simple square plate geometry. Recently, Kawabata et al., [13] conducted wind tunnel experiments to investigate the influence of the downstream strip plate length on the phenomena reported by Koide et al., [11]. The results are summarized as follows: KVIV is less suppressed when the plate length $l_{d}$ is smaller and almost no-suppression occurs at $I_{d} / D=1$ (square plate). The galloping is effectively suppressed by short strip plates by setting the gap ratio smaller when the strip plate is shorter, e.g. $S / D<2$ for $I_{d} / D=1$. Although the LVIV does not occur when $I d / D<4$, a large amplitude vibration was found to be induced by short strip plates, i.e. $I_{d} / D=1$ and 2 when $S / D \leq 1$. Since this vibration is considered to be caused by the fluid elastic instability, they called it "wake body interference fluid elastic vibration (WBIFEV)".

The abovementioned findings by Kawabata et al., [13] regarding the influence of the downstream square plate on the vibration of the square cylinder can potentially lead to a novel technique to control FIVs of cylindrical bodies, which is not only to avoid structural damage but also to be utilized for beneficial purposes such as electricity generation. However, their experiment was carried out for limited conditions and the influences on KVIV and galloping were investigated by varying the gap at respective velocities of KVIV and galloping. Therefore, the objectives of this study are: 
i. To investigate the influence of the downstream square plate on the cross-flow vibration of square cylinder by performing wind tunnel experiments under comprehensive conditions in which vibration response against varying flow velocity is measured for fixed values of $S / D$.

ii. To identify the effect of gap conditions on vibration behaviour to develop a novel passive technique to induce or to suppress the vibration.

\section{Experimental Apparatus, Instruments, Procedure and Parameters of Vibrating System}

Experiments were conducted in the test section connected to a blow-down type wind tunnel in the mechanical laboratory of the Malaysian Japan Higher Education Project (MJHEP), Beranang, Malaysia. The test section has a $0.36 \mathrm{~m} \times 0.36 \mathrm{~m}$ cross section, which is identical with the outlet nozzle section of the wind tunnel, and a $0.8 \mathrm{~m}$ length. The walls of the test section are made of transparent acrylic plates to record the cylinder oscillation using a camera. Figure 1 shows the outline of the experimental apparatus and setting of instruments, together with the coordinate system $O-x$, $y, z$ used in this paper. Note that the origin ' $O$ ' is set at the centre of the square cylinder. The rigid square cylinder models are made from acrylic resin plate with a side length $D=26 \mathrm{~mm}$ and a length $L=351.4 \mathrm{~mm}$. The cylinder is fixed to a supporting rod passing through the slots on the side walls of the test section and supported by springs outside the test section. Circular disks made of acrylic plate with a thickness $1.2 \mathrm{~mm}$ are attached to the cylinder at both ends to prevent air flow through the slots. The length of the cylinder $L$ is defined as the distance between the two end disks. The twinplate springs ensure the oscillation in a purely cross-flow mode. The magnetic dampers are mounted on the frame structure at both sides of the cylinder to support the rod outside the measuring section. The damping factor is adjusted by moving the magnets mounted on the traversing device (Kawabata et al., [13], Marra et al., [19], Mannini et al., [20-21], etc). The downstream square plate is made of spring steel plate with a thickness of $5 \mathrm{~mm}$ and has a side length $w$ equal to that of the cylinder, i.e. $w=26 \mathrm{~mm}$. The gap $S$ between the cylinder and the plate is adjusted in the range of $S / D=0.12 \sim$ 2.90 .

The flow velocity $U$ is measured by a Pitot tube (JIS type, $\varnothing 9 \times(L) 0.5 \mathrm{~m}$ ) connected to a pressure gauge (Manostar, Type W081, Range: 0-100Pa \& 0-500Pa). A hot wire anemometer (Dantec Dynamics, Probe type $55 \mathrm{P} 11$ ) is used to detect the $x$-component of velocity at a point in the near wake, i.e. $x=2 D, y=D$ and $z=1.5 D$. The vortex shedding frequency $f_{v}$ is determined from the peak frequency in the spectrum of the velocity signal $u$ detected by the hot wire anemometer. A laser displacement sensor (Omron ZX-LDA11) is used to measure the vertical displacement of cylinder $Y$. The oscillation amplitude is determined as the root mean square value of $Y$, and the oscillation frequency $f_{c}$ is determined from the peak frequency in the spectrum of $Y$. The data processing for $u$ and $Y$ is carried out using the LabVIEW program after converting them into digital signals with NI USB9215. The data acquisition system has four channels with a sampling rate of $100 \mathrm{kHz} / \mathrm{ch}$ and a resolution of 16 bit in analogue-to-digital converters (ADCs). 


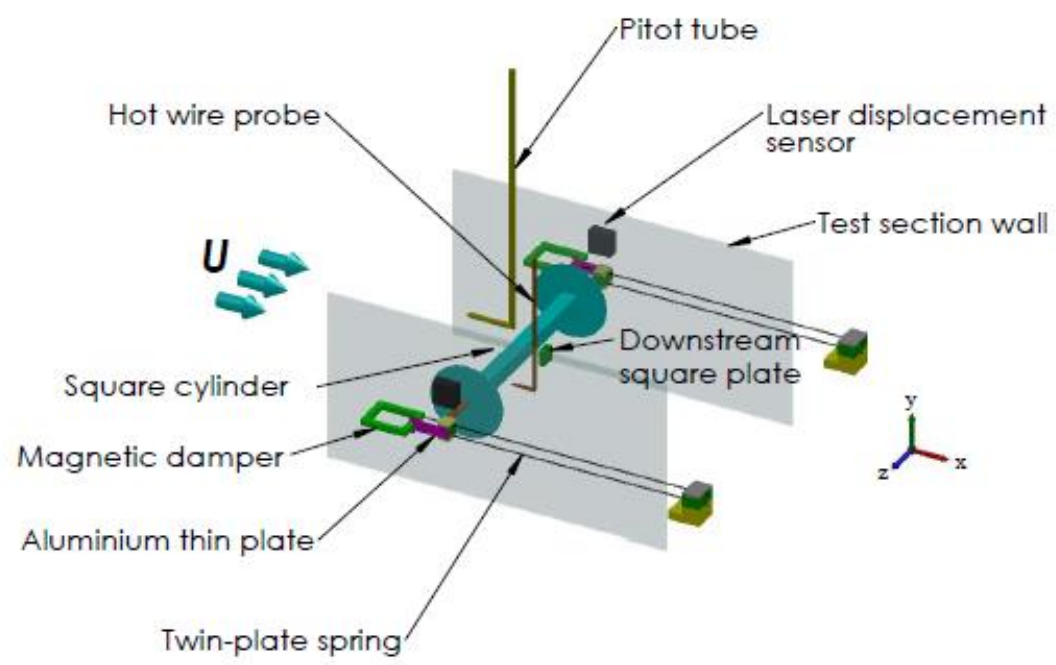

Fig. 1. Schematic diagram of experimental apparatus and instruments, together with the coordinate system used in this paper. $U$ is the flow velocity

The natural frequency and the logarithmic damping factor are determined from the displacement signal $Y$ of the free damping oscillation at $U=0$. That is, the time interval $T_{m}$ for $m$-cycles of $Y$ is measured and $f_{n}$ is determined as $f_{n}=m / T_{m}$, which is fixed at $16.5 \mathrm{~Hz}$ and equal to that of Kawabata et al., [13]. The logarithmic damping factor $\delta$ is determined by using Eq. (1):

$\delta=(1 / m) \ln \left(Y_{1} / Y_{(m+1)}\right)$,

where $Y_{1}$ is the peak amplitude in the first cycle and $Y_{(m+1)}$ is that of $(m+1)$-th cycle. The value of $\delta$ is also adjusted to make the value of critical reduced velocity of galloping $U_{g}$ approximately equal to that of Kawabata et al., [13]. Then, the effective mass $m_{e}$ is calculated by

$m_{e}=k /\left(2 \pi f_{n}\right)^{2}$,

where $k$ is the total spring constant of the supporting system measured separately. The mass ratio, i.e. the ratio of the effective mass to the mass of fluid displaced by the cylinder, is defined as $M_{R}=$ $m_{e} /\left(\rho D^{2} L\right)$. Scruton number, Sc defined by Eq. (3) represents the coupled effect of the mass and the damping and considered to be the dominating parameter for the oscillation response (see e.g. Kawabata et al., [13] or Kaneko et al., [22]).

$S c=2 M_{R} \delta$

The conditions, specification of the cylinder system and the non-dimensional parameters obtained from the above procedure are shown in Table 1, together with those of other studies for comparison.

Firstly, the vibration amplitude $Y_{r m s}$, the vibration frequency $f_{c}$ and the vortex shedding frequency $f_{v}$ against flow velocity $U$ were measured for the isolated square cylinder. The velocity range was from the velocity lower than KVIV until the full galloping velocity. Then, the influence of the square plate on the relationships between $Y_{r m s}, f_{c}, f_{v}$ and $U$ was measured for various values of $S$. The results of $Y_{r m s}, f_{c}, f_{v}$ are properly normalised and plotted against the reduced velocity $U_{R}$ defined by Eq. (4).

$U_{R}=U /\left(f_{n} D\right)$ 


\section{Table 1}

Conditions and parameters of studies compared in Figure 2. (Wind tunnel experiment: The present study, Ismail et al., [23] and Kawabata et al., [13]; Numerical simulation: Maruai et al., [4])

\begin{tabular}{|c|c|c|c|c|}
\hline Parameter & Present & Maruai & Ismail & Kawabata \\
\hline $\begin{array}{l}\text { Square cylinder side length, } D \\
(\mathrm{~mm})\end{array}$ & 26 & 26 & 26 & 26 \\
\hline Length of cylinder, $L(\mathrm{~mm})$ & 351.4 & 315 & 315 & 315 \\
\hline Kinematic viscosity of air, $v\left(\mathrm{~m}^{2} / \mathrm{s}\right)$ & $1.55 \times 10^{-5}$ & $1.54 \times 10^{-5}$ & $1.54 \times 10^{-5}$ & $1.56 \times 10^{-5}$ \\
\hline Air density, $\rho\left(\mathrm{kg} / \mathrm{m}^{3}\right)$ & 1.134 & 1.201 & 1.204 & 1.157 \\
\hline Natural frequency, $f_{n}(\mathrm{~Hz})$ & 16.5 & 14.3 & 14.3 & 16.5 \\
\hline Spring constant, $k(\mathrm{~N} / \mathrm{m})$ & 2495 & 1173 & 1173 & 1181 \\
\hline Logarithmic damping factor, $\delta$ & 0.018 & 0.027 & 0.027 & 0.027 \\
\hline Effective mass, $m_{e}(\mathrm{~kg})$ & 0.23 & 0.15 & 0.15 & 0.11 \\
\hline Mass ratio, $M_{R}$ & 867.58 & 566.74 & 565.22 & 446.30 \\
\hline Scruton number, Sc & 31.75 & 31.17 & 31.09 & 24.10 \\
\hline Reynolds number, $R e$ & $3.9-14.8 \times 10^{3}$ & $4.2-10.7 \times 10^{3}$ & $3.67-11.73 \times 10^{3}$ & $3.8-16.2 \times 10^{3}$ \\
\hline Reduced velocity, $U_{R}$ & $5.5-20.6$ & $5-20$ & $5.7-18.2$ & $5.3-19.5$ \\
\hline
\end{tabular}

\section{Results and Discussions}

\subsection{Vibration Behaviour of The Isolated Square Cylinder}

Figure 2 shows the results of the present experiments for the isolated cylinder, which are compared with the previous studies, i.e. the experiments by Kawabata et al., [13] and Ismail et al., [23], and the simulation study by Maruai et al., [4] as shown in Table 1. In Figure 2(a), the nondimensional amplitude is multiplied by $S c$ and plotted against the reduced velocity $U_{R}$ for fair comparison since the maximum KVIV amplitude would be inversely proportional with $S c$ if the vibration system were linear. The vortex shedding frequency $f_{v}$ and the vibration frequency $f_{c}$ are determined as the highest peak frequency of spectra $S_{u}$ and $S_{Y}$ respectively as shown in Figure 3 . They are normalized by the natural frequency $f_{n}$ and plotted against $U_{R}$ in Figure 2(b) and Figure 2(c).

The tendency of the amplitude response of the present work agrees well with all the other studies as shown in Figure 2(a), and this can be described as follows: 1) The velocity ranges of KVIV and galloping are separated by essentially zero vibration range. 2) KVIV occurs in the velocity range around the velocity at which the natural vortex shedding frequency $f_{v o}$ is equal to the natural frequency $f_{n}$, although the maximum KVIV amplitude peak $A_{K V I V}$ is significantly scattered among the studies even when it is normalized by multiplying Sc. 3) The critical reduced velocity of galloping $U_{g}$, i.e. onset of vibration due to galloping, coincides well, but the increasing gradient after $U_{g}$ is different among the studies. Ismail et al., [23] mentioned that the second peak value of around $U_{R}=10.5$ from the result of Kawabata et al., [13] was due to the rolling motion of the cylinder due to un-symmetry of the cylinder structure. This vibration is properly erased in the present study.

The vibration at velocities lower than the KVIV range is the forced vibration excited by the alternating lift of natural vortex shedding. That is, the amplitude remains small, and $f_{v}$ increases linearly with $U$ while $f_{c}=f_{v}<f_{n}$ as seen in Figure 2. Similar to the studies by Khalak and Williamson [24] and Feng [25] for a circular cylinder, the vibration amplitude starts increasing at $U_{R}$ at which the natural vortex shedding frequency $f_{v o}$ is lower than the natural frequency $f_{n}$. The KVIV regime is confirmed by seeing whether the Karman vortex synchronizes with the cylinder oscillation. When the synchronization occurs, the spectrum $S_{u}$ has a sharp peak at the oscillation frequency $f_{c}$ which is nearly equal to $f_{n}$ as seen in Figure 3(a). The maximum KVIV amplitude $A_{K V I V}$ appears at around the critical reduced velocity at which $f_{v o}=f_{n}$. The KVIV regime persists over a certain range of velocity until the desynchronization and the drop of vibration amplitude occur showing the end of KVIV. In 
many cases, this regime near the higher velocity end of KVIV is unstable in the sense that the KVIV and the small vibration-desynchronization regimes are irregularly alternating.

The vibration in the intermediate range is also the forced vibration excited by the natural vortex shedding. As a result, $f_{v}$ increases linearly with $U$ and $f_{c}=f_{v}$, being higher than $f_{n}$. However, the present study shows that $f_{c} / f_{n}=1$ in the range of $8.5 \leq U_{R} \leq 15.3$ as shown in Figure 2(c). This paradoxical result is caused by the selective resonance explained as follows.

When the exciting force has a widely distributing spectrum, the vibration response will also have a wide spectrum in which every frequency component is amplified according to the theory of vibration. Therefore, when the damping factor is small, the component of the exciting force at the frequency equal to the natural frequency $f_{n}$ causes very large vibration at $f=f_{n}$. This phenomenon is called "selective resonance". As a result, the spectrum of displacement can have a peak at $f_{n}$ higher than the peak at the exciting force frequency. In the present study, this occurs in the intermediate region, as clearly observed in Figure $3(\mathrm{~b})$ for $U_{R}=10.93$, making $f_{c} / f_{n}=1$ since the oscillation frequency $f_{c}$ is defined as the highest peak frequency in $S_{Y}$.

The amplitude abruptly starts increasing at $U_{R}=15.8$ with the frequency $f_{c}=f_{n}$, while the vortex shedding frequency $f_{v}$ remains equal to $f_{v o}$ as shown in Figure 2 . Hence, this is identified as the critical reduced velocity of galloping $U_{g}$ (Sun et al., [26]). In the full galloping regime, $S_{Y}$ has a very high sharp peak at $f_{n}$, and the peak at $f_{v}$ due to the selective resonance disappears as seen in Figure $3(c)$. The galloping regime persists up to the maximum $U_{R}$ of measurement of the present experiment. The agreement of the value of $U_{g}$ is excellent among the studies, but the increasing gradient of vibration amplitude is different. Although the values of Scruton number $S c$ in Table 1 are nearly equal among the researches compared in Figure 2, the amplitude normalized by Sc is significantly different each other. This result confirms that the mass ratio and the damping factor influence the amplitude response independently (Nguyen et al., [8]; Mannini et al., [20]; Sun et al., [26]).

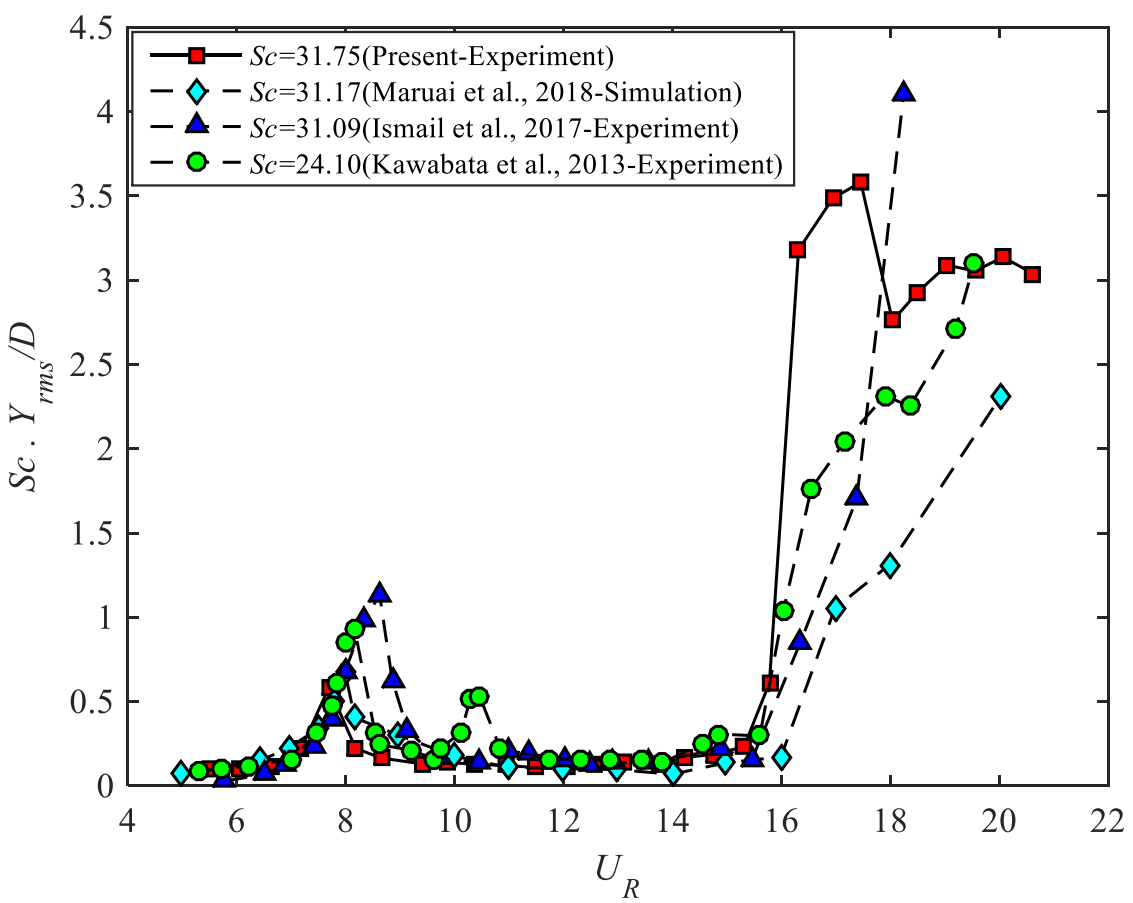

(a) 


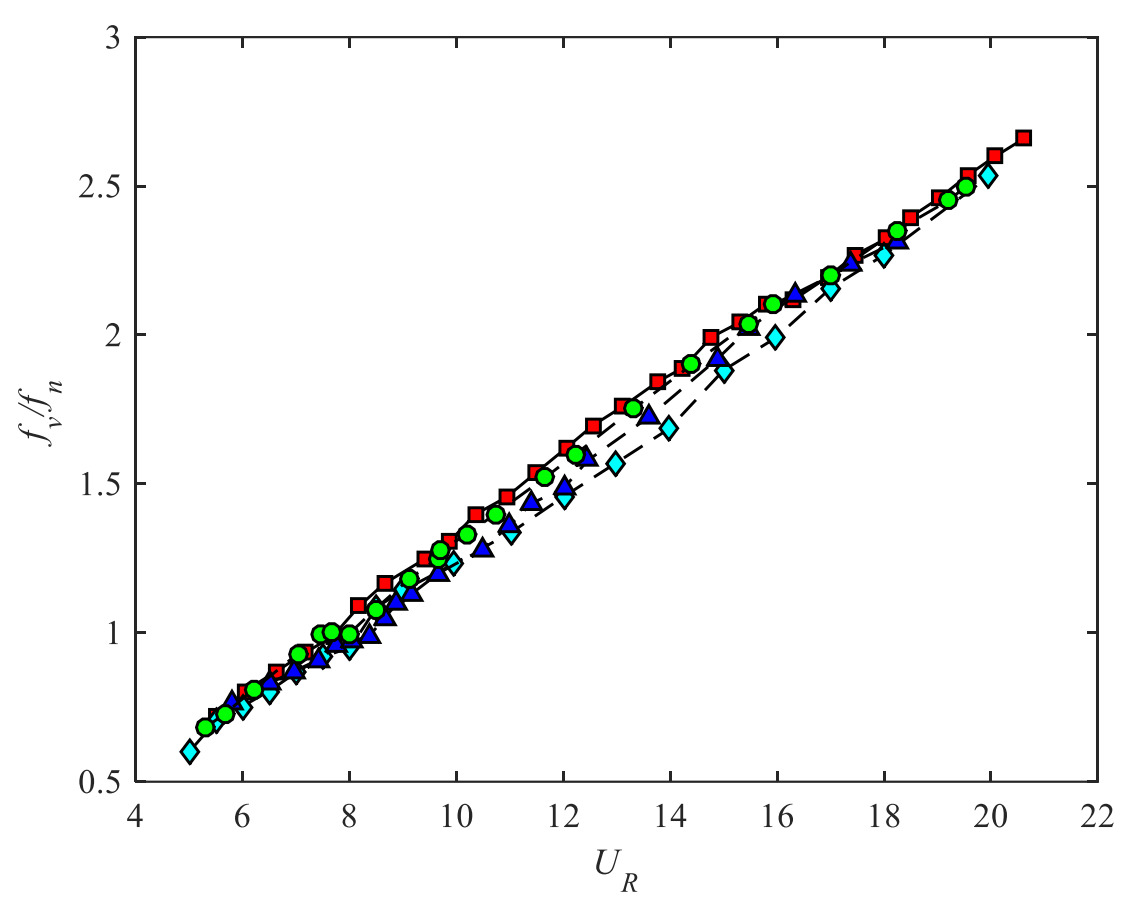

(b)

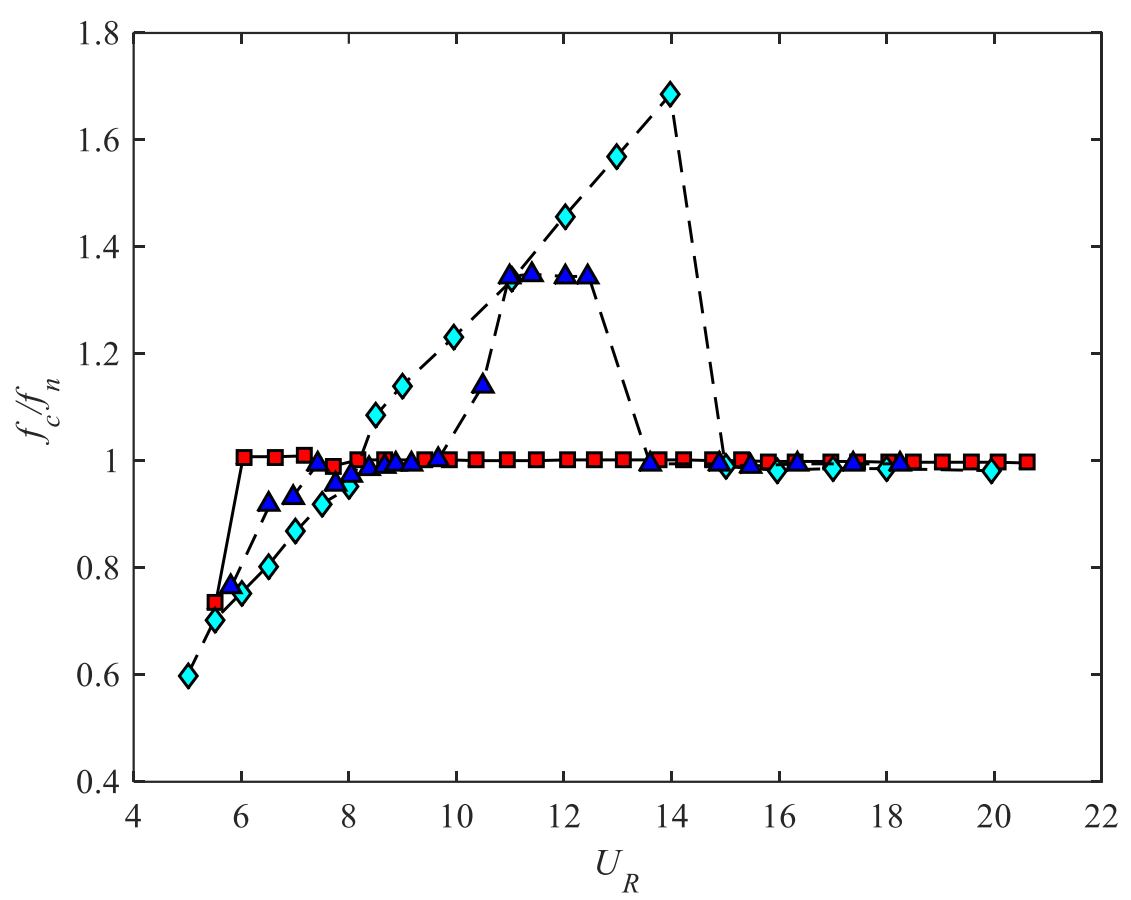

(c)

Fig. 2. Vibration and vortex shedding behaviours for the isolated cylinder, compared with previous studies i.e. the simulation study by Maruai et al., [4] and the experimental studies by Ismail et al., [23] and Kawabata et al., [13] 


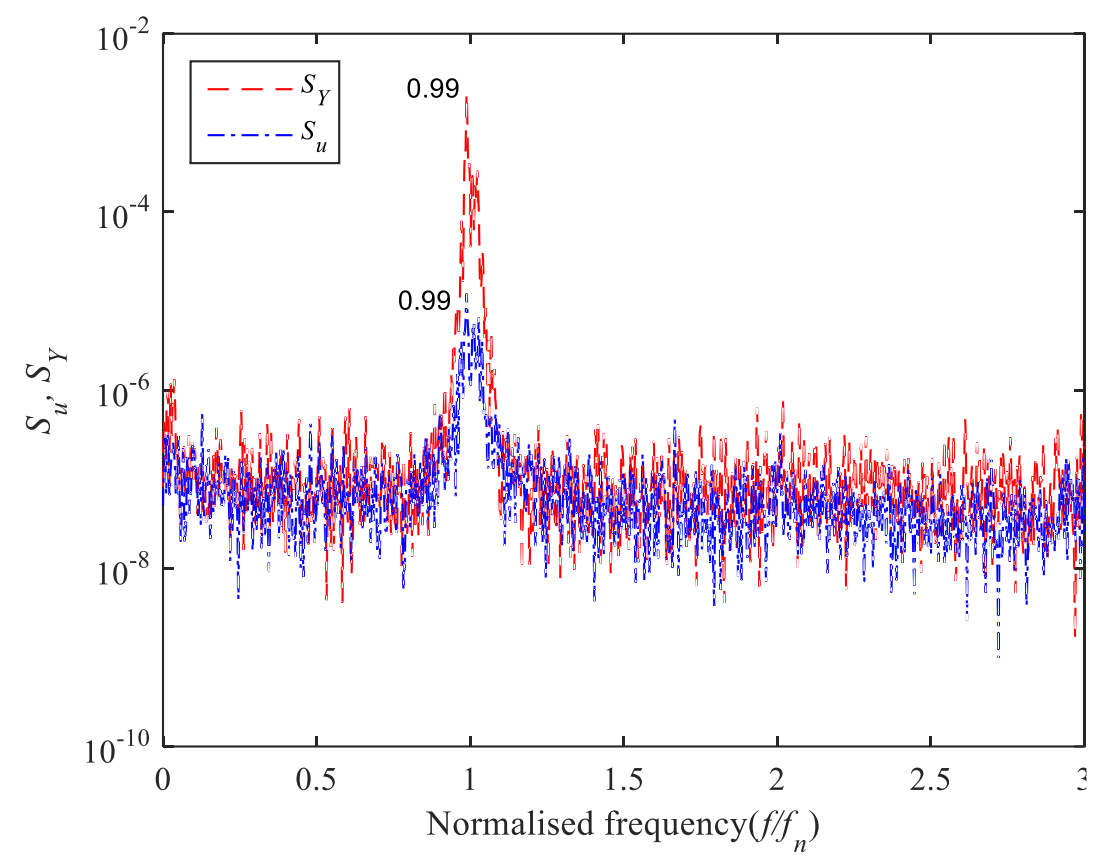

(a)

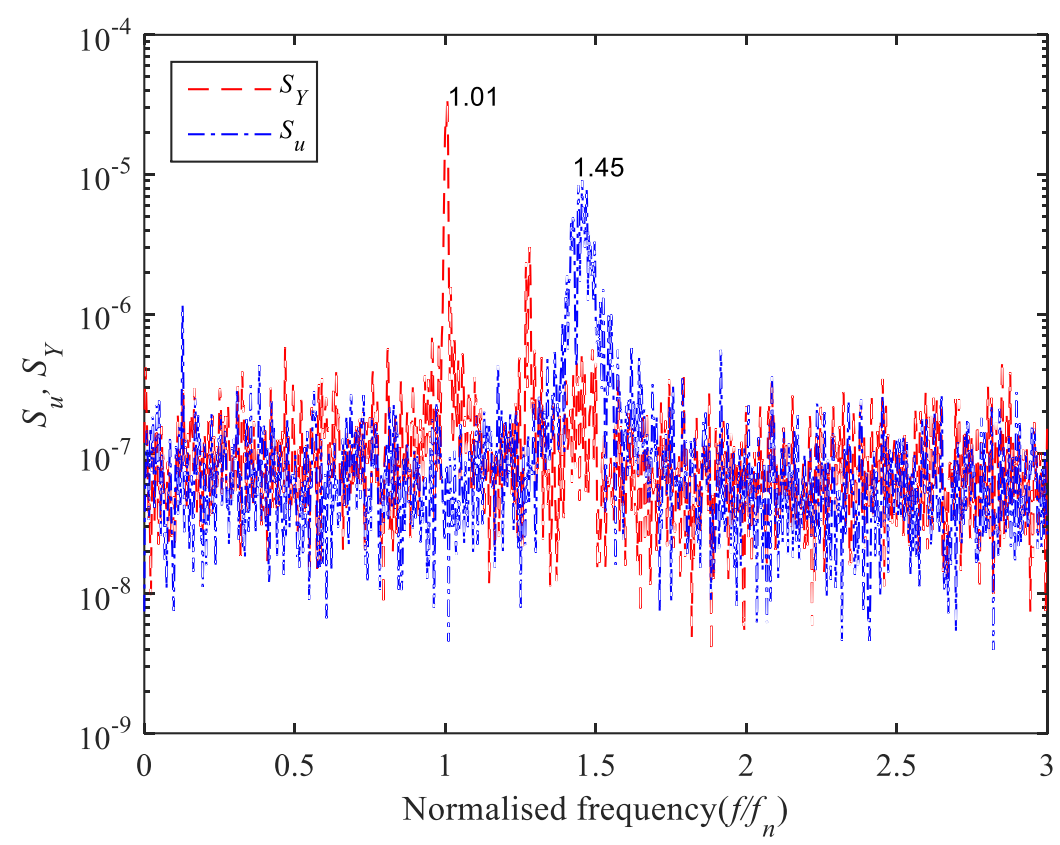

(b) 


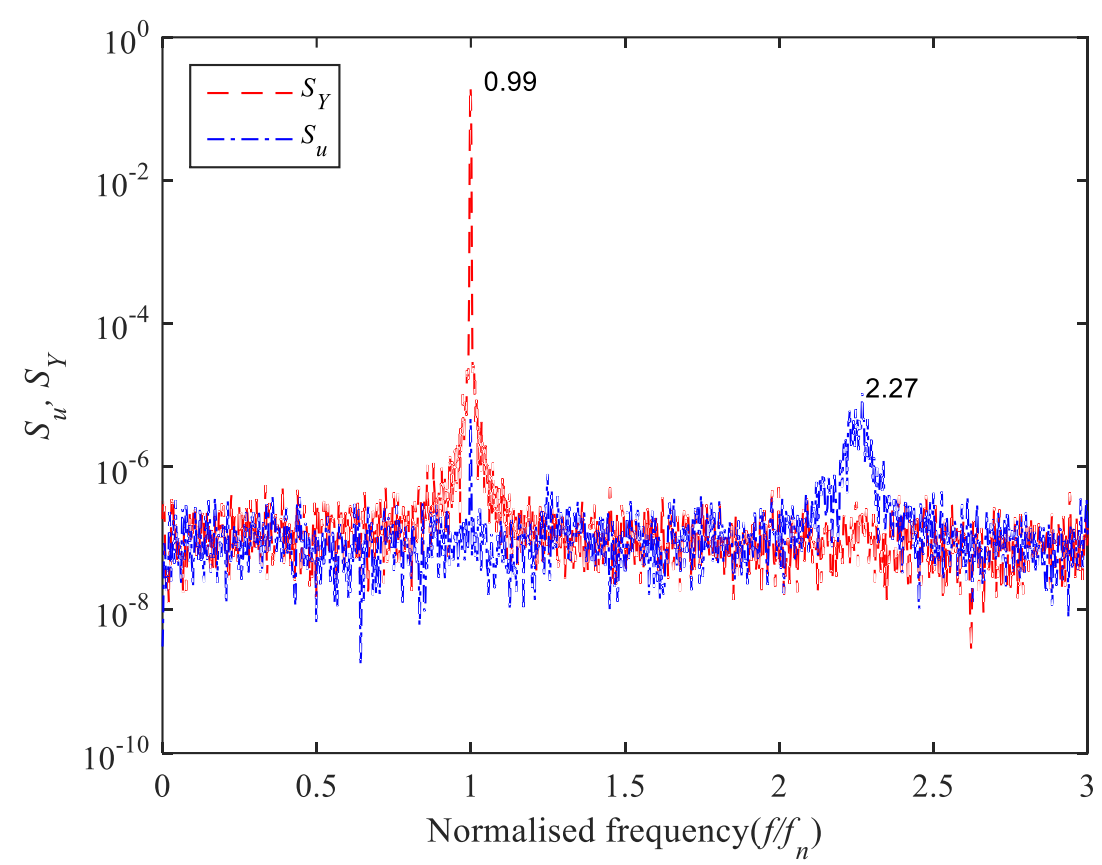

(c)

Fig. 3. Power Spectrum Density (PSD) of displacement $Y$ and velocity $u$. (Isolated square cylinder, $S c=31.75, S_{y}$ : red line, $S_{u}$ : blue line): (a) $U_{R}=7.69\left(f_{d} / f_{n}=0.99, f_{v} / f_{n}=0.99\right) ;\left(\right.$ b) $U_{R}=10.93\left(f_{d} / f_{n}=1.00, f_{v} / f_{n}=\right.$ 1.45); (c) $U_{R}=17.46\left(f_{d} / f_{n}=0.99, f_{v} / f_{n}=2.27\right)$

\subsection{Effect of The Downstream Square Plate}

In Figure 4, the non-dimensional amplitude is plotted against $U_{R}$ for various gap ratios $S / D$ over a range from 0.12 to 2.90 . To see the effect of the downstream plate on KVIV, the maximum amplitude $A_{K V I V}$ and the reduced velocity $U_{A}$ for $A_{K V I V}$ are obtained from the results in Figure 4 . and plotted against $S / D$ in Figure 5 . Figure 5 shows that $A_{K V I V} / D$ and $U_{A}$ are only slightly affected in the range of gap ratio $S / D \leq 0.98$. At $S / D=1.17, A_{K V I V} / D$ reaches the largest value, around five times larger and $U_{A}$ increases higher by $20 \%$, as compared with the isolated counterpart. They decrease with $S / D$ in the range of $1.17 \leq S / D \leq 2.33$ and return to their respective values of the isolated counterpart at $S / D=$ 2.90. This result is consistent with that of Kawabata et al., [13] which reported that the influence of downstream square cylinder on KVIV is insignificant, except the large vibration observed at $S / D=$ 1.17 .

The influence of the downstream plate on the galloping observed in Figure 4 can be summarized as follows: i) When $S / D \geqq 1.75$, the influence of the downstream plate is small, ii) When $1.37 \geqq S / D$ $\geqq 1.17$, the increasing gradient of amplitude after $U_{g}$ decreases with the decreasing gap ratio while $U_{g}$ is not affected, iii) When $0.98 \geqq S / D \geqq 0.12, U_{g}$ is much smaller than that of the isolated counterpart while the increasing gradient of amplitude after $U_{g}$ is almost un-affected. In order to compare with Kawabata et al., [13], the vibration amplitude in the full galloping regime, i.e. at $U_{R}=$ 18.4 indicated by the vertical red broken line in Figure $4(\mathrm{~b})$, is obtained and normalized by that without the downstream plate, defined as $Y_{r m s} / Y_{r m s \infty}$. It is plotted against $S / D$ in Figure $6(a)$ and compared with the results of Kawabata et al., [13] together with $U_{g}$ vs. $S / D$ as shown in Figure $6(b)$. Since in Figure $4(\mathrm{a})$ the data of the present study at $U_{R}=18.4$ is not available for $S / D<1.17$, the amplitude at the maximum $U_{R}$ of measurement in the galloping regime is regarded to be equivalent, and this is also plotted in Figure 6(a). 
In contrast to the findings of Kawabata et al., [13] the downstream square plate does not suppress, but rather enhances the galloping as seen in Figure 6 . That is, the critical reduced velocity $U_{g}$ is lower than that of the isolated counterpart when $S / D \leqq 2.13$, and, generally speaking, decreases with decreasing $S / D$. However, $U_{g}$ is minimum, around half of the isolated counterpart, at $S / D=0.98$. The galloping amplitude which is slightly suppressed in the range of $1.17 \leq S / D \leq 1.94$ is considered to be caused by the slow gradient of galloping amplitude after $U_{g}$. At the present stage, the reason for this inconsistency is quite unclear thus becomes the topic of further investigation.

From the viewpoint of physics of fluid motion, it is questionable to identify the large amplitude vibration at $U_{R}=9.88$ for $S / D=1.17$ shown in Figure 4(b) as the KVIV is enhanced by the downstream plate, and because its maximum amplitude is around five times larger than that of KVIV of the isolated counterpart. It is not plausible that the small downstream plate can enhance the excitation force of KV so largely. The spectra of $Y$ and $u$ at this vibration are presented in Figure 7. By comparing this figure with Figure 3(a), it seems that the synchronization of KV on the cylinder oscillation is not so firm. Hence, this phenomenon may be regarded as the "Wake body interference fluid elastic vibration (WBIFEV)" as found by Kawabata et al., [13].

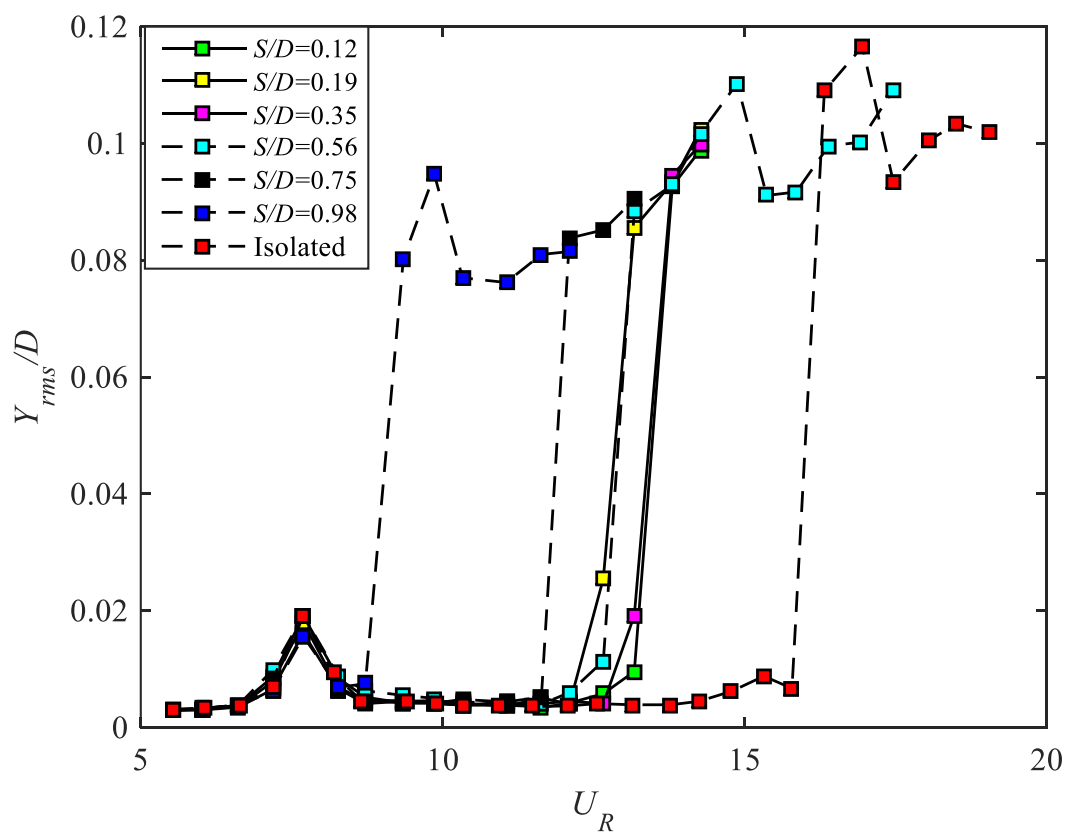

(a) 


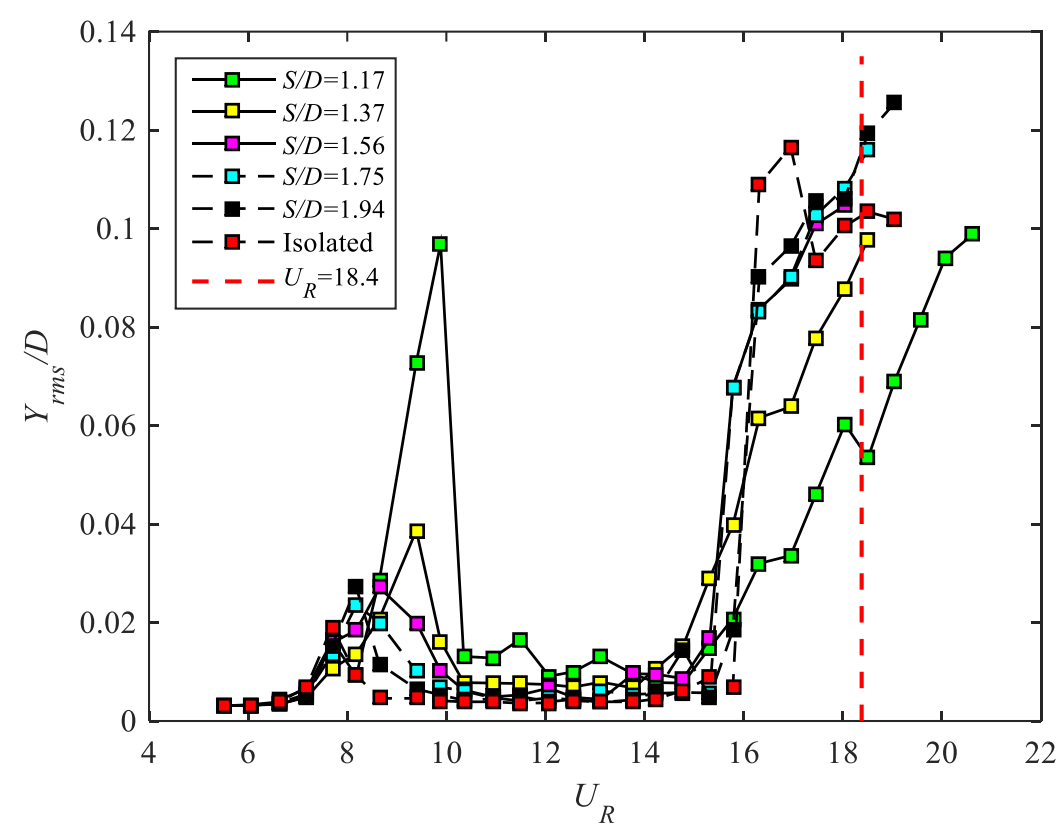

(b)

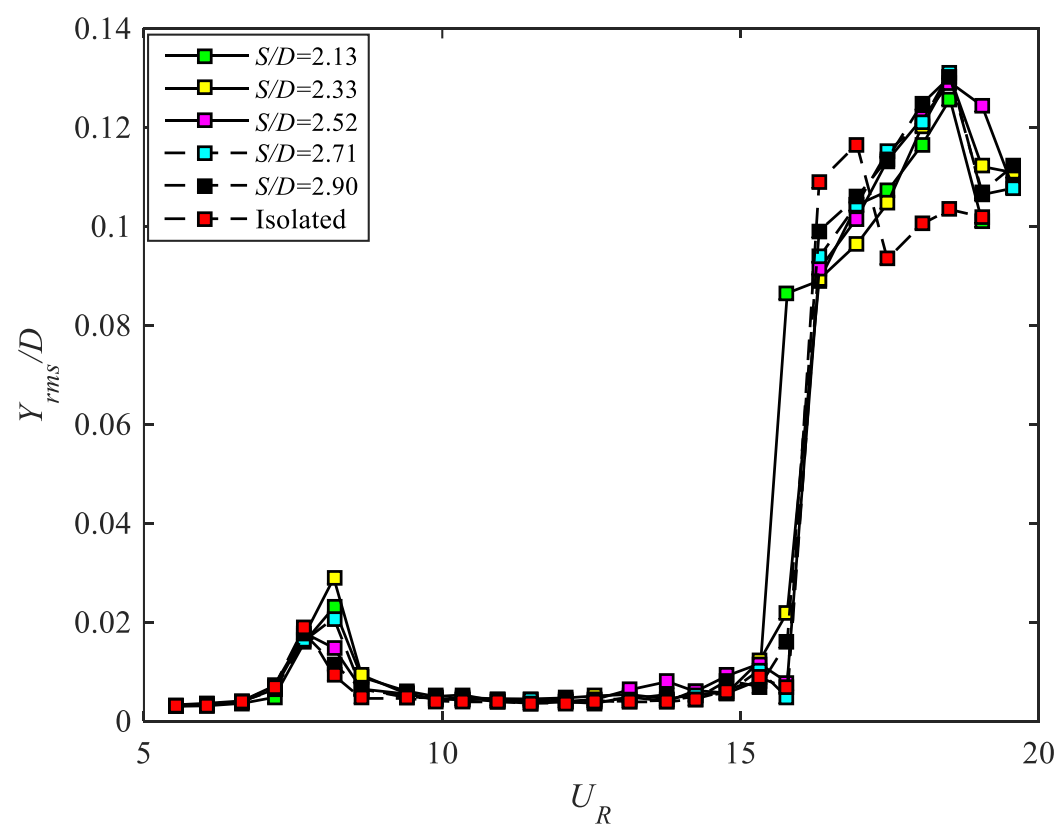

(c)

Fig. 4. Influence of square plate $(w / D=1)$ on relationship between vibration amplitude and flow velocity of the square cylinder. (a) 0.12 $\leq S / D \leq 0.98$; (b) $1.17 \leq S / D \leq 1.94$; (c) $2.13 \leq S / D \leq 2.90$. The vertical red broken line in Figure $4(\mathrm{~b})$ at $U_{R}=18.38$ indicates the reduced velocity in the experiment by Kawabata et al., [13] for varying $S / D$ 


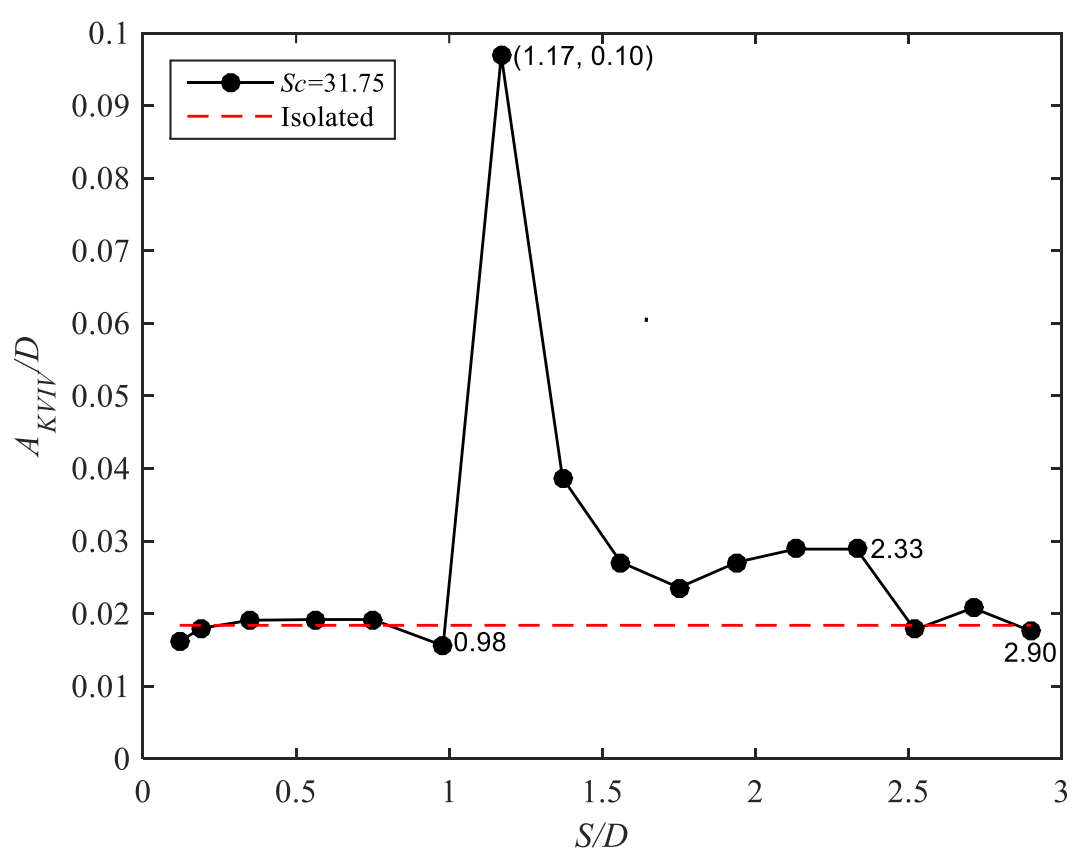

(a)

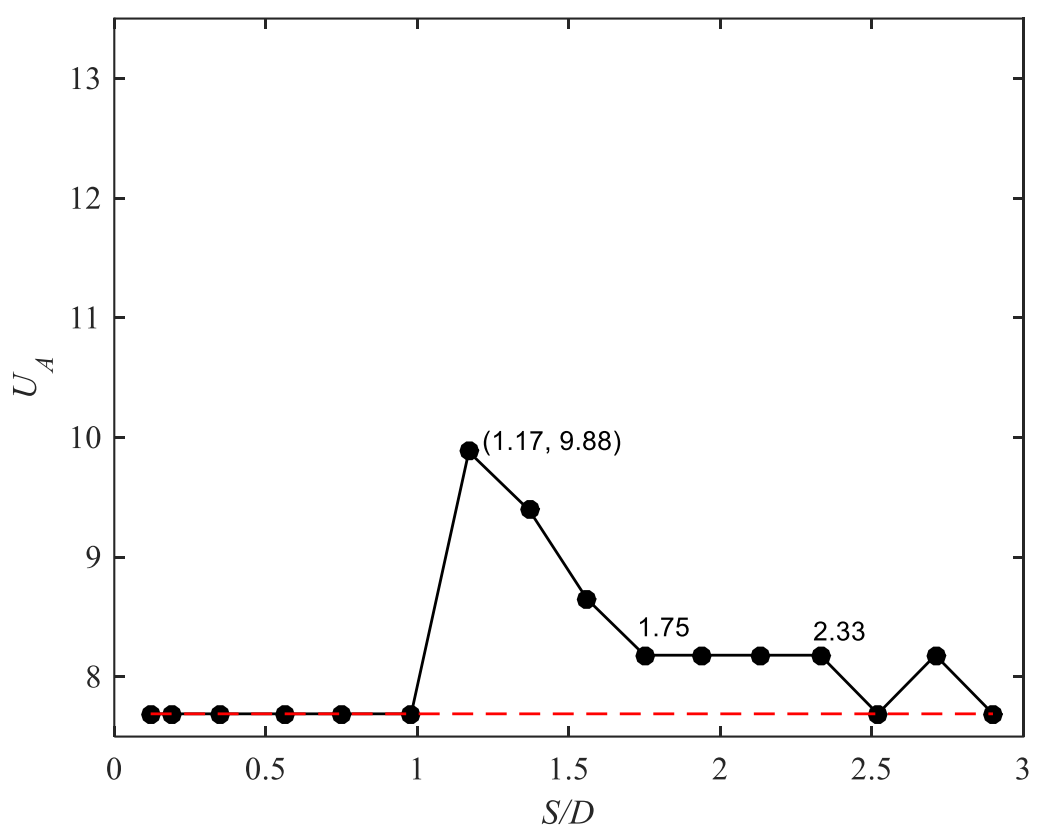

(b)

Fig. 5. The influence of square plate $\left(I_{d} / D=w / D=1\right)$ on KVIV of the square cylinder: (a) Maximum amplitude of KVIV, (b) Reduced velocity at maximum amplitude of KVIV. The horizontal red broken line represents the case of the isolated counterpart 


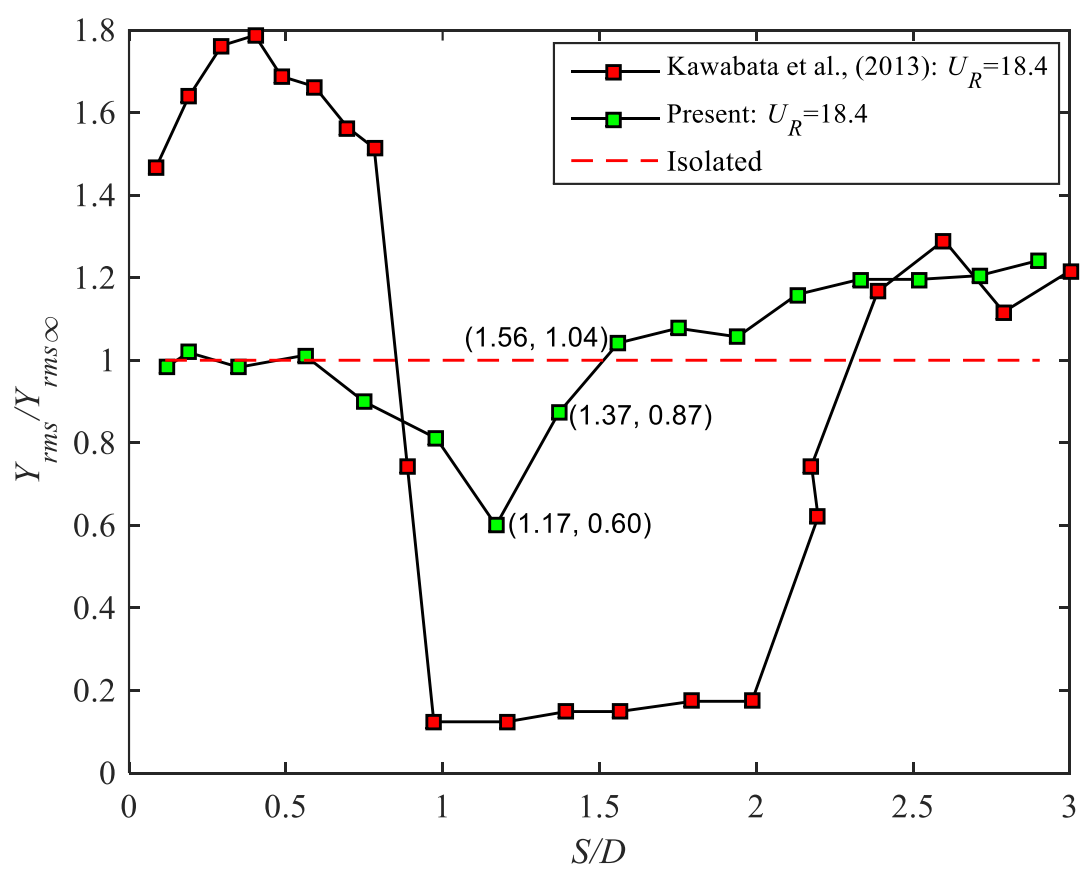

(a)

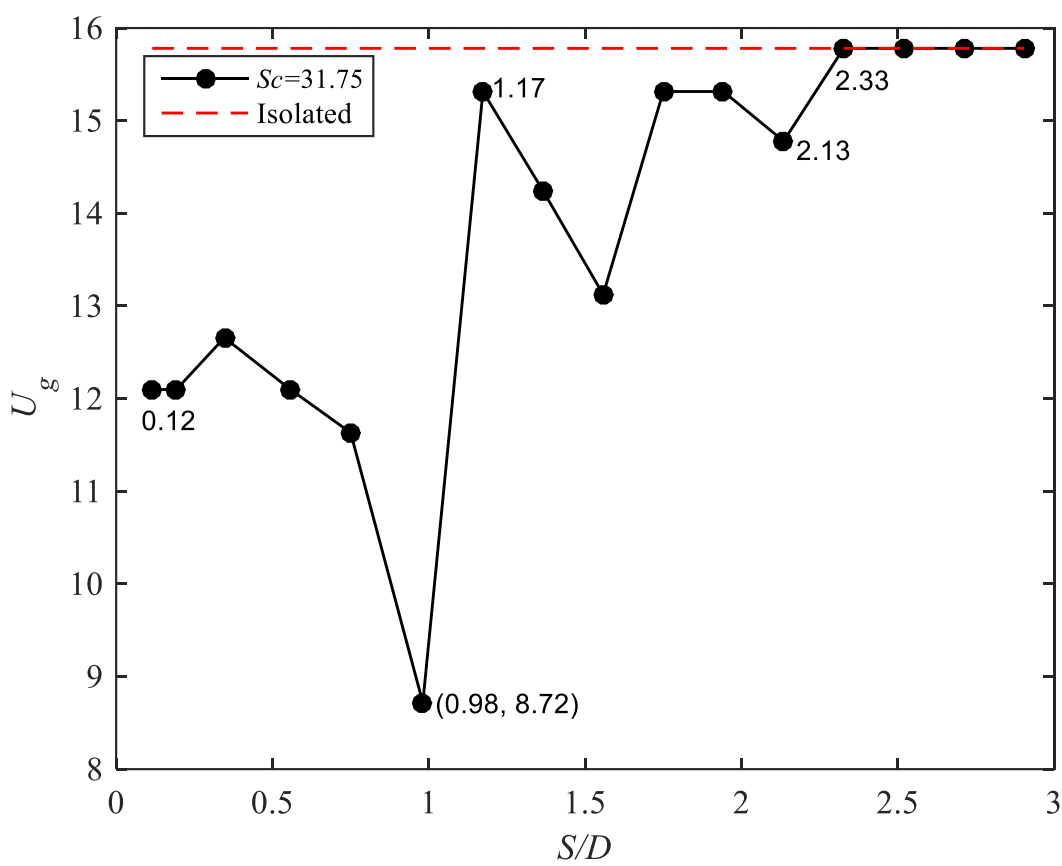

(b)

Fig. 6. The influence of square plate $(w / D=1)$ on galloping of the square cylinder compared with Kawabata et al., [13]: (a) Galloping amplitude normalized by that without downstream plate at $U_{R}=18.4$, (b) Critical reduced velocity of galloping $U_{g}$. The horizontal red broken line represents the case of the isolated counterpart 


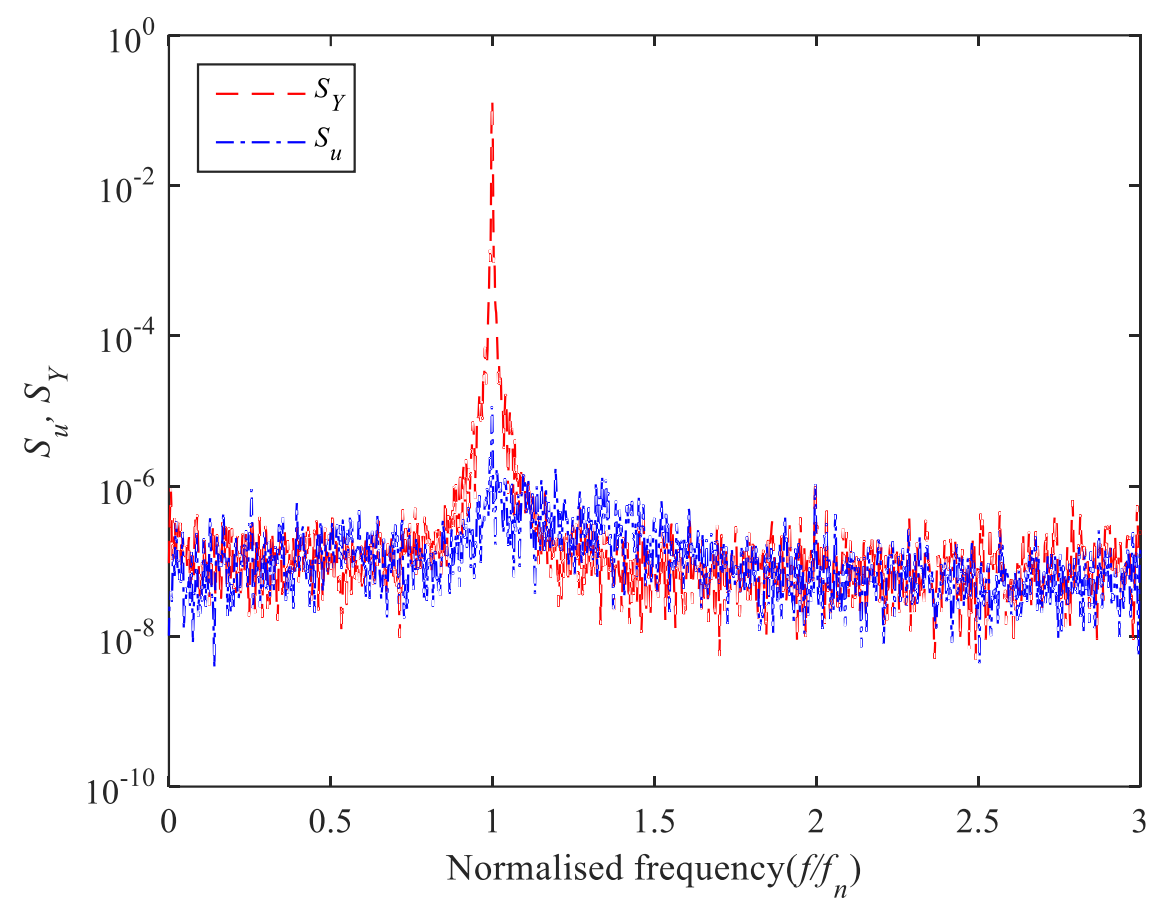

Fig. 7. Power Spectrum Density $S_{Y}$ and $S_{u}$ at $U_{R}=9.88$ for the maximum KVIV peak with the downstream plate placed at $S / D=1.17$

\section{Conclusions}

The present study aims to develop a passive technique to control cross-flow vibrations of a square cylinder using a downstream square plate. The square plate which has a side length equal to that of the square cylinder is most simple and desirable as the downstream body from the practical point of view. The results of wind tunnel experiment for the isolated square cylinder are in good agreement with the previous experiments and simulations for the amplitude response against varying velocity, which validated the experimental facilities/techniques in the present study.

The downstream square plate is shown to only have a slight effect on KVIV as reported by Kawabata et al., [13]. In contrast, it enhances the galloping in the sense that the onset velocity of galloping reduces to about half of the isolated counterpart at $S / D=0.98$. The most remarkable effect of the downstream square plate is the enhancement of the vibration near the peak velocity of KVIV at $S / D=1.17$. This may indicate the occurrence of the wake-body-interference fluid elastic vibration reported by Kawabata et al., [13]. The effect of the downstream square plate on the square cylinder found in this study will lead to a novel technique for vibration control. However, more detailed and comprehensive experiments are expected to clarify the cause of difference with respect to the findings of Kawabata et al., [13].

\section{Acknowledgement}

This research was supported by the Malaysian Ministry of Higher Education (MoHE) under the Fundamental Research Grant Scheme (5F150) project of Universiti Teknologi Malaysia.

\section{References}

[1] Terres-Nicoli, J. M., and Gregory A. Kopp. "Mechanisms of the vertical vortex induced vibration of the Storebælt Bridge." In Proceedings of the 11th Americas Conference on Wind Engineering, San Juan, Puerto Rico, pp. 22-26. 2009.

[2] Assi, Gustavo RS, P. W. Bearman, and N. Kitney. "Low drag solutions for suppressing vortex-induced vibration of circular cylinders." Journal of Fluids and Structures 25, no. 4 (2009): 666-675. 
https://doi.org/10.1016/j.jfluidstructs.2008.11.002

[3] Ali, Mohamed Sukri Mat, Con J. Doolan, and Vincent Wheatley. "Low Reynolds number flow over a square cylinder with a detached flat plate." International Journal of Heat and Fluid Flow 36 (2012): 133-141.

https://doi.org/10.1016/i.ijheatfluidflow.2012.03.011

[4] Maruai, Nurshafinaz Mohd, Mohamed Sukri Mat Ali, Mohamad Hafiz Ismail, and Sheikh Ahmad Zaki. "Flow-induced vibration of a square cylinder and downstream flat plate associated with micro-scale energy harvester." Journal of Wind Engineering and Industrial Aerodynamics 175 (2018): 264-282.

https://doi.org/10.1016/i.jweia.2018.01.010

[5] Dash, Sunil Manohar, Michael S. Triantafyllou, and Pablo Valdivia Y. Alvarado. "A numerical study on the enhanced drag reduction and wake regime control of a square cylinder using dual splitter plates." Computers \& Fluids (2019): 104421.

https://doi.org/10.1016/i.compfluid.2019.104421

[6] Shirakashi, M., K. Mizuguchi, and H. M. Bae. "Flow-induced excitation of an elastically-supported cylinder caused by another located downstream in cruciform arrangement." Journal of Fluids and Structures 3, no. 6 (1989): 595607.

https://doi.org/10.1016/S0889-9746(89)90150-3

[7] Bae, Heon Meen, László Baranyi, Mizuyasu Koide, Tsutomu Takahashi, and Masataka Shirakashi. "Suppression of Kármán vortex excitation of a circular cylinder by a second cylinder set downstream in cruciform arrangement." Journal of Computational and Applied Mechanics 2, no. 2 (2001): 175-188.

[8] Nguyen, T., M. Koide, S. Yamada, T. Takahashi, and M. Shirakashi. "Influence of mass and damping ratios on VIVs of a cylinder with a downstream counterpart in cruciform arrangement." Journal of fluids and structures 28 (2012): 40-55.

https://doi.org/10.1016/i.jfluidstructs.2011.10.006

[9] Ma, Yexuan, Wanhai Xu, Libin Zhai, and Huanan Ai. "Hydrodynamic characteristics of two tandem flexible cylinders undergoing flow-induced vibration." Ocean Engineering 193 (2019): 106587. https://doi.org/10.1016/i.oceaneng.2019.106587

[10] Chauhan, Manish Kumar, Sushanta Dutta, and Bhupendra Kumar Gandhi. "Wake flow modification behind a square cylinder using control rods." Journal of Wind Engineering and Industrial Aerodynamics 184 (2019): 342-361. https://doi.org/10.1016/j.jweia.2018.12.002

[11] Koide, M., Kato, N., Yamada, S., Kawabata, Y., Takahashi, T., Shirakashi, M. "Influence of a cruciform arrangement downstream strip-plate on crossflow vibration of a square cylinder." Journal of Computational and Applied mechanics 8, no. 2 (2007): 135-148.

[12] Kato, Naoto, Mizuyasu Koide, Tsutomu Takahashi, and Masataka Shirakash. "VIVs of a circular cylinder with a downstream strip-plate in cruciform arrangement." Journal of fluids and structures 30 (2012): 97-114. https://doi.org/10.1016/i.jfluidstructs.2012.02.007

[13] Kawabata, Yusuke, Tsutomu Takahashi, Takeshi Haginoya, and Masataka Shirakashi. "Interference effect of downstream strip-plate on the crossflow vibration of a square cylinder." Journal of Fluid Science and Technology 8 , no. 3 (2013): 348-363.

https://doi.org/10.1299/ifst.8.348

[14] Assi, Gustavo RS, Peter W. Bearman, and Michael A. Tognarelli. "On the stability of a free-to-rotate short-tail fairing and a splitter plate as suppressors of vortex-induced vibration." Ocean engineering 92 (2014): 234-244. https://doi.org/10.1016/i.oceaneng.2014.10.007

[15] Yu, Yue, Fangfang Xie, Hongmei Yan, Yiannis Constantinides, Owen Oakley, and George Em Karniadakis. "Suppression of vortex-induced vibrations by fairings: a numerical study." Journal of Fluids and Structures 54 (2015): 679-700. https://doi.org/10.1016/i.jfluidstructs.2015.01.007

[16] Wang, Jiasong, Hanxu Zheng, and Zhongxu Tian. "Numerical simulation with a TVD-FVM method for circular cylinder wake control by a fairing." Journal of Fluids and Structures 57 (2015): 15-31. https://doi.org/10.1016/j.jfluidstructs.2015.05.008

[17] Lou, Min, Wu-gang $\mathrm{Wu}$, and Peng Chen. "Experimental study on vortex induced vibration of risers with fairing considering wake interference." International Journal of Naval Architecture and Ocean Engineering 9, no. 2 (2017): $127-134$.

[18] Zheng, Hanxu, and Jiasong Wang. "Galloping oscillation of a circular cylinder firmly combined with different shaped fairing devices." Journal of Fluids and Structures 77 (2018): 182-195. https://doi.org/10.1016/i.jfluidstructs.2017.12.010 
[19] Marra, Antonino Maria, Claudio Mannini, and Gianni Bartoli. "Measurements and improved model of vortexinduced vibration for an elongated rectangular cylinder." Journal of Wind Engineering and Industrial Aerodynamics 147 (2015): 358-367. https://doi.org/10.1016/i.jweia.2015.08.007

[20] Mannini, C., A. M. Marra, and G. Bartoli. "VIV-galloping instability of rectangular cylinders: Review and new experiments." Journal of wind engineering and industrial aerodynamics 132 (2014): 109-124. https://doi.org/10.1016/i.jweia.2014.06.021

[21] Mannini, Claudio, Antonino Maria Marra, Tommaso Massai, and Gianni Bartoli. "Interference of vortex-induced vibration and transverse galloping for a rectangular cylinder." Journal of Fluids and Structures 66 (2016): $403-423$. https://doi.org/10.1016/i.jfluidstructs.2016.08.002

[22] Shigehiko Kaneko, Nakamura, Tomomichi, Fumio Inada, Minoru Kato, Kunihiko Ishihara, Takashi Nishihara, Njuki W. Mureithi, and Mikael A. Langthjem, eds. Flow-induced vibrations: classifications and lessons from practical experiences. Butterworth-Heinemann, 2013.

[23] Ismail, Mohamad Hafiz, Mohamed Sukri Mat Ali, Sheikh Ahmad Zaki Shaikh Salim, Masataka Shirakashi, and Sallehuddin Muhamad. "FLOW INDUCED VIBRATION OF A SQUARE CYLINDER WITH HIGH SCRUTON NUMBER." ARPN J. of Eng. and Applied Sciences 12, no. 10 (2017).

[24] Khalak, A., and Charles HK Williamson. "Motions, forces and mode transitions in vortex-induced vibrations at low mass-damping." Journal of fluids and Structures 13, no. 7-8 (1999): 813-852. https://doi.org/10.1006/jfls.1999.0236

[25] FENG, CC. "The measurement of vortex-induced effects in flow past stationary and oscillating circular and D-Section cylinders." MASc thesis, University of British (1968).

[26] Sun, Hai, Eun Soo Kim, Gary Nowakowski, Erik Mauer, and Michael M. Bernitsas. "Effect of mass-ratio, damping, and stiffness on optimal hydrokinetic energy conversion of a single, rough cylinder in flow induced motions." Renewable Energy 99 (2016): 936-959.

https://doi.org/10.1016/i.renene.2016.07.024 